\title{
A CRITERION FOR POTENTIALLY GOOD REDUCTION IN NON-ARCHIMEDEAN DYNAMICS
}

\author{
ROBERT L. BENEDETTO
}

\begin{abstract}
Let $K$ be a non-archimedean field, and let $\phi \in K(z)$ be a polynomial or rational function of degree at least 2 . We present a necessary and sufficient condition, involving only the fixed points of $\phi$ and their preimages, that determines whether or not the dynamical system $\phi: \mathbb{P}^{1} \rightarrow \mathbb{P}^{1}$ has potentially good reduction.
\end{abstract}

Fix the following notation throughout this paper.

$\begin{array}{ll}K: & \text { a field } \\ \bar{K}: & \text { an algebraic closure of } K \\ |\cdot|_{v}: & \text { a non-archimedean absolute value on } \bar{K} \\ \mathcal{O}_{K}: & \text { the ring of integers }\left\{x \in K:|x|_{v} \leq 1\right\} \text { of } K \\ \mathcal{M}_{K}: & \text { the maximal idea }\left\{x \in K:|x|_{v}<1\right\} \text { of } \mathcal{O}_{K} \\ k: & \text { the residue field } \mathcal{O}_{K} / \mathcal{M}_{K} \text { of } K \\ \mathcal{O}_{\bar{K}}: & \text { the ring of integers of } \bar{K} \\ \mathcal{M}_{\bar{K}}: & \text { the maximal idea of } \mathcal{O}_{\bar{K}} \\ \bar{k}: & \text { the residue field of } \bar{K}\end{array}$

For example, $K$ could be the field $\mathbb{Q}_{p}$ of $p$-adic rationals, with ring of integers $\mathbb{Z}_{p}$, maximal ideal $p \mathbb{Z}_{p}$, and algebraic closure $\overline{\mathbb{Q}}_{p}$. Since $\mathbb{Q}_{p}$ is complete, the absolute value $|\cdot|_{p}$ on $\mathbb{Q}_{p}$ extends uniquely to $\overline{\mathbb{Q}}_{p}$.

Let $\phi(z) \in K(z)$ be a rational function. We define the degree of $\phi=f / g$ to be $\operatorname{deg} \phi:=\max \{\operatorname{deg} f, \operatorname{deg} g\}$, where $f, g \in K[z]$ have no common factors. We will view $\phi$ as a a dynamical system acting on $\mathbb{P}^{1}(\bar{K})=\bar{K} \cup\{\infty\}$. For a thorough treatment of dynamics over such non-archimedean fields, see [1].

If $\operatorname{deg} \phi \geq 2$, then $\phi$ fixes exactly $1+\operatorname{deg} \phi$ points in $\mathbb{P}^{1}(\bar{K})$, counted with appropriate multiplicity. The multiplier $\lambda \in \bar{K}$ of such a fixed

Date: November 30, 2013.

2010 Mathematics Subject Classification. Primary: 37P05 Secondary: 37P20, $11 \mathrm{~S} 82$.

Key words and phrases. arithmetic dynamics, good reduction, periodic points. 
point $x \in \mathbb{P}^{1}(\bar{K})$ is

$$
\lambda:= \begin{cases}\phi^{\prime}(x) & \text { if } x \in \bar{K}, \\ (\psi)^{\prime}(0) & \text { if } x=\infty, \text { where } \psi(z)=1 / \phi(1 / z) .\end{cases}
$$

If $|\lambda|_{v}>1$, we say the fixed point $x$ is repelling; if $|\lambda|_{v}=1$, we say $x$ is indifferent; and if $|\lambda|_{v}<1$, we say $x$ is attracting. The multiplier of $x$ is exactly 1 (as opposed to merely having absolute value 1 ) if and only if $x$ has multiplicity at least two as a fixed point. It is 0 if and only if $x$ is a critical point of $\phi$.

If we change coordinates on $\mathbb{P}^{1}(\bar{K})$ by applying a linear fractional transformation $h \in \operatorname{PGL}(2, \bar{K})$, the effect on $\phi$ is to conjugate by $h$. In particular, if $x$ is a fixed point of $\phi$, then $h(x)$ is a fixed point of $h \circ$ $\phi \circ h^{-1} \in \bar{K}(z)$, and with the same multiplier. This invariance justifies the definition of the multiplier of a fixed point at $\infty$ in equation (0.1).

The notion of good reduction of $\phi \in K(z)$ was first stated in [4]; see Definition 1.1. We will say that $\phi$ has potentially good reduction if $\phi$ is conjugate over $\bar{K}$ to a map of good reduction. It is easy to see whether or not a given map $\phi \in K(z)$ has good reduction as written, but in general, it is not so obvious how to tell whether or not $\phi$ is conjugate to a map of good reduction.

In [6], Rumely presents two somewhat involved algorithms for deciding whether or not $\phi$ has potentially good reduction. A much simpler necessary and sufficient condition for potential good reduction when $\phi \in K[z]$ is a polynomial appeared in Corollary 4.6 of [2]. In this paper, we generalize that simpler criterion to apply to arbitrary rational functions $\phi \in K(x)$, as follows.

Theorem. Let $K$ be a field with algebraic closure $\bar{K}$ and with nonarchimedean absolute value $|\cdot|_{v}$ on $\bar{K}$. Let $\phi \in K(z)$ be a rational function of degree $d \geq 2$. Let $x_{1}, \ldots, x_{d+1} \in \mathbb{P}^{1}(\bar{K})$ be the fixed points of $\phi$, repeated according to multiplicity. If any $x_{i}$ is repelling, then $\phi$ does not have potentially good reduction. Otherwise, define $h \in \operatorname{PGL}(2, \bar{K})$ as follows.

(1) If $x_{i}$ is indifferent for some $i$, then it is possible to choose $y_{1} \in$ $\phi^{-1}\left(x_{i}\right) \backslash\left\{x_{i}\right\}$, and $y_{2} \in \phi^{-1}\left(y_{1}\right)$. The three points $x_{i}, y_{1}, y_{2} \in$ $\mathbb{P}^{1}(\bar{K})$ are necessarily distinct, and so there is a unique $h \in$ $\operatorname{PGL}(2, \bar{K})$ such that $h\left(x_{i}\right)=0, h\left(y_{1}\right)=1$, and $h\left(y_{2}\right)=\infty$.

(2) Otherwise, all the fixed points are attracting, and $x_{1}, x_{2}$, and $x_{3}$ are necessarily distinct. Thus, there is a unique $h \in \operatorname{PGL}(2, \bar{K})$ such that $h\left(x_{1}\right)=0, h\left(x_{2}\right)=1$, and $h\left(x_{3}\right)=\infty$. 
Let $\psi=h \circ \phi \circ h^{-1}$. The original map $\phi$ has potentially good reduction if and only if $\psi$ has good reduction; in that case, $h$ provides the desired coordinate change.

If $\phi$ does have potentially good reduction, the map $h \in \operatorname{PGL}(2, \bar{K})$ of the above Theorem is defined over an extension of degree at most $d^{3}-d$ over $K$, a priori. After all, the fixed points of $\phi$ are all roots of a polynomial of degree $d+1$, and the preimages of any given point of $\mathbb{P}^{1}(\bar{K})$ are roots of a polynomial of degree $d$. In case (a) above, then, $x_{i}$ contributes degree up to $d+1, y_{1}$ contributes up to $d-1$, and $y_{2}$ contributes up to $d$. Similarly, in case (b), $x_{0}, x_{1}$, and $x_{2}$ contribute up to $d+1, d$, and $d-1$. However, in a separate paper [3], we will show that this bound may be reduced from $d^{3}-d$ to $\max \left\{d+1, d^{2}-d\right\}$.

We will recall some general facts about good reduction in Section 1 . Then, in Section 2, we will state some simple lemmas and prove the Theorem.

\section{REDUCTION OF RATIONAL MAPS}

Given a polynomial $f(z) \in \mathcal{O}_{\bar{K}}[z]$, denote by $\bar{f}(z) \in \bar{k}[z]$ the polynomial formed by reducing all coefficients of $f$ modulo $\mathcal{M}_{\bar{K}}$.

Definition 1.1. Let $\phi(z) \in K(z)$ be a rational function of degree $d \geq 1$. Write $\phi=f / g$ with $f, g \in \mathcal{O}_{K}[z]$ and with at least one coefficient of $f$ or $g$ having absolute value 1 . Let $\bar{\phi}:=\bar{f} / \bar{g}$. We say that $\phi$ has $g$ ood reduction if $\operatorname{deg} \bar{\phi}=\operatorname{deg} \phi$. Otherwise, we say that $\phi$ has bad reduction.

We say that $\phi$ has potentially good reduction if there is some $h \in$ $\operatorname{PGL}(2, \bar{K})$ such that $h \circ \phi \circ h^{-1} \in \bar{K}(z)$ has good reduction.

There is some inconsistency in the literature as to whether "bad reduction" of a dynamical system $\phi$ should mean the lack of good reduction, or, more restrictively, the lack of good reduction even after a $K$ rational change of coordinates. For example, if $a \in K$ is a uniformizer, then the map $\phi(z)=a z^{2}$ has bad reduction as written, but its $K$ rational conjugate $a \phi\left(a^{-1} z\right)=z^{2}$ has good reduction. Meanwhile, the map $\psi(z)=a z^{3}$ also has bad reduction, and in fact it can be shown to have bad reduction even after any $K$-rational coordinate change. However, $\psi$ has potentially good reduction, because $a^{1 / 2} \psi\left(a^{-1 / 2} z\right)=z^{3}$ has good reduction.

Given $\phi \in K(z)$, it is easy to check that polynomials $f, g \in \mathcal{O}_{K}[z]$ exist as specified in Definition 1.1. and that the reduction type of $\phi$ does not change if two other such polynomials $\tilde{f}, \tilde{g} \in \mathcal{O}_{K}[z]$ are chosen in their stead. Note that $\operatorname{deg} \bar{\phi} \leq \operatorname{deg} \phi$, but the degree could drop if the formerly relatively prime $f, g \in \mathcal{O}_{K}[z]$ gain a common factor 
when they are reduced, or if $\max \{\operatorname{deg} \bar{f}, \operatorname{deg} \bar{g}\}$ is strictly smaller than $\max \{\operatorname{deg} f, \operatorname{deg} g\}$.

The reduction map red $: \mathcal{O}_{\bar{K}} \rightarrow \bar{k}$ induces a map red $: \mathbb{P}^{1}(\bar{K}) \rightarrow$ $\mathbb{P}^{1}(\bar{k})$, which coincides with the original reduction map on $\mathcal{O}_{\bar{K}}$ and maps $\mathbb{P}^{1}(\bar{K}) \backslash \mathcal{O}_{\bar{K}}$ to the point $\bar{\infty} \in \mathbb{P}^{1}(\bar{k})$. It is easy to check that a rational function $\phi \in \bar{K}(z)$ has good reduction if and only if it respects the reduction map, i.e., if

$$
\bar{\phi}(\bar{x})=\overline{\phi(x)} \quad \text { for all } \quad x \in \mathbb{P}^{1}(\bar{K}) .
$$

As a result, the composition of two maps of good reduction again has good reduction. In addition, among maps of degree 1, i.e., in $\operatorname{PGL}(2, \bar{K})$, the good reduction maps are precisely the elements of $\operatorname{PGL}\left(2, \mathcal{O}_{\bar{K}}\right)$.

Lemma 1.2. Let $\psi \in \bar{K}(z)$ have good reduction, and write $\psi=f / g$ with $f, g \in \mathcal{O}_{\bar{K}}[z]$ as in Definition 1.1. Suppose that 0 is a fixed point of $\psi$, and let $\lambda$ be the associated multiplier. Then $f(0)=0,|g(0)|_{v}=1$, and $|\lambda|_{v}=\left|f^{\prime}(0)\right|_{v}$.

Proof. Since $f$ and $g$ are relatively prime, we have $f(0)=0$ and $g(0) \neq$ 0 . However, because $\bar{f}, \bar{g} \in \mathcal{O}_{\bar{K}}[z]$ are also relatively prime, we must in fact have $|g(0)|_{v}=1$. Thus,

$$
|\lambda|_{v}=\left|\frac{f^{\prime}(0) g(0)-f(0) g^{\prime}(0)}{g(0)^{2}}\right|_{v}=\frac{\left|f^{\prime}(0) g(0)\right|_{v}}{|g(0)|_{v}^{2}}=\left|f^{\prime}(0)\right|_{v} .
$$

\section{Proof of the TheOREM}

The following lemmas are well known in the more general setting of dynamics on a non-archimedean disk, but their proofs in our context are very short and simple, and so we include them for the convenience of the reader.

Lemma 2.1. Let $\psi \in \bar{K}(z)$ be a rational function of good reduction, and let $x \in \mathbb{P}^{1}(\bar{K})$ be a fixed point. Then $x$ is indifferent or attracting.

Proof. There is some degree one map $h \in \mathrm{PGL}\left(2, \mathcal{O}_{\bar{K}}\right)$ of good reduction such that $h(x)=0$. Since conjugating by $h$ preserves good reduction and multipliers, we may assume without loss that $x=0$. Let $\lambda:=\psi^{\prime}(0)$ be the associated multiplier.

Write $\psi=f / g$ as in Definition [1.1. Then $f^{\prime} \in \mathcal{O}_{\bar{K}}[z]$, since $f \in$ $\mathcal{O}_{\bar{K}}[z]$. Thus, by Lemma 1.2 , we have $|\lambda|_{v}=\left|f^{\prime}(0)\right|_{v} \leq 1$.

Lemma 2.2. Let $\psi \in \bar{K}(z)$ be a rational function of good reduction, and let $x \in \mathbb{P}^{1}(\bar{K})$ be an attracting fixed point of $\psi$. Then for any other 
fixed point $y \in \mathbb{P}^{1}(\bar{K})$ of $\psi$, the reductions $\operatorname{red}(x), \operatorname{red}(y) \in \mathbb{P}^{1}(\bar{k})$ are distinct.

Proof. By a PGL $\left(2, \mathcal{O}_{\bar{K}}\right)$ change of coordinates, we may assume that $x=0$. Write $\psi=f / g$ as in Definition 1.1, and let $\lambda:=\psi^{\prime}(0)$ be the associated multiplier. By Lemma 1.2, we have $f(0)=0,|g(0)|_{v}=1$, and $\left|f^{\prime}(0)\right|_{v}=|\lambda|_{v}<1$. That is, $f(z)=a_{1} z+\cdots+a_{d} z^{d}$ with $\left|a_{i}\right|_{v} \leq 1$ and $\left|a_{1}\right|_{v}<1$, whereas $g(z)=b_{0}+b_{1} z+\cdots+b_{d} z^{d}$ with $\left|b_{i}\right|_{v} \leq 1$ and $\left|b_{0}\right|_{v}=1$.

For any $y \in \mathbb{P}^{1}(\bar{K})$ with $\operatorname{red}(y)=\operatorname{red}(0)$, we have $|y|_{v}<1$. Thus, if $y \neq 0$, we have

$|\psi(y)|_{v}=|y|_{v} \cdot \frac{\left|a_{1}+\cdots+a_{d} y^{d-1}\right|_{v}}{\left|b_{0}+\cdots+b_{d} y^{d}\right|_{v}}=|y|_{v} \cdot \frac{\left|a_{1}+\cdots+a_{d} y^{d-1}\right|_{v}}{\left|b_{0}\right|_{v}}<|y|_{v}$,

and hence $y$ cannot be fixed.

Lemma 2.3. Let $\psi \in \bar{K}(z)$ be a rational function of good reduction and degree $d \geq 2$, and let $x \in \mathbb{P}^{1}(\bar{K})$ be an indifferent fixed point of $\psi$. Then $\psi^{-1}(x) \backslash\{x\}$ is nonempty, and $\operatorname{red}(x) \neq \operatorname{red}(y)$ for any $y \in \psi^{-1}(x) \backslash\{x\}$.

Proof. By a $\mathrm{PGL}\left(2, \mathcal{O}_{\bar{K}}\right)$ change of coordinates, we may assume that $x=0$. Write $\psi=f / g$ as in Definition 1.1, and let $\lambda:=\psi^{\prime}(0)$ be the associated multiplier. By Lemma [1.2, we have $f(0)=0,|g(0)|_{v}=1$, and $\left|f^{\prime}(0)\right|_{v}=|\lambda|_{v}=1$. Thus, $f(z)=a_{1} z+\cdots+a_{d} z^{d}$ with $\left|a_{i}\right|_{v} \leq 1$ and $\left|a_{1}\right|_{v}=1$. It is immediate that $f$, and hence $\psi$, has no roots in $\mathcal{M}_{\bar{K}} \backslash\{0\}$.

Meanwhile, the hypothesis on $\psi^{\prime}(0)$ also implies that 0 is not a critical point. Since $d \geq 2, \psi^{-1}(0) \backslash\{0\}$ is nonempty. Given $y \in \psi^{-1}(0) \backslash$ $\{0\}$, the previous paragraph shows that $y \notin \mathcal{M}_{\bar{K}}$, and hence $\operatorname{red}(y) \neq$ $\operatorname{red}(0)$.

Remark 2.4. Lemmas 2.2 and 2.3 are special cases more general statements about fixed points of a map $\phi \in K(z)$ of good reduction. Specifically, if $x$ is an attracting fixed point (or more generally, periodic point) of such a map, then the residue class of $x$ contains no other periodic points, although it almost certainly contains many other preperiodic points that ultimately map to $x$ itself. Similarly, if $x$ is an indifferent periodic point of a map of good reduction, then the residue class of $x$ maps bijectively onto itself, and hence the only preperiodic points in the residue class are in fact periodic. (In fact, there are usually infinitely many periodic points in such a residue class.)

Furthermore, these facts about preperiodic points near nonrepelling periodic points were generalized by Rivera-Letelier in his thesis [5] to 
maps of bad reduction, by replacing residue classes by an appropriate notion of Fatou components. However, we will only need the far simpler statements of Lemmas 2.2 and 2.3 here.

Proof of the Theorem. For each $i=1, \ldots, d+1$, let $\lambda_{i} \in \bar{K}$ be the multiplier of the fixed point $x_{i}$. If $\phi$ has potentially good reduction, then there is some $h \in \operatorname{PGL}(2, \bar{K})$ such that $h \circ \phi \circ h^{-1}$ has good reduction. The fixed points of $h \circ \phi \circ h^{-1}$ have the same multipliers as those of $\phi$, and therefore, by Lemma 2.1, we have $\left|\lambda_{i}\right|_{v} \leq 1$ for all $i$, proving the first statement of the Theorem. We now consider two cases.

Case 1. If $\left|\lambda_{i}\right|_{v}=1$ for some $i$, then the associated fixed point $x_{i}$ is not a critical point (since $\lambda_{i} \neq 0$ ), and hence $\phi^{-1}\left(x_{i}\right) \backslash\left\{x_{i}\right\}$ is nonempty. Pick $y_{1} \in \phi^{-1}\left(x_{i}\right) \backslash\left\{x_{i}\right\}$, and pick $y_{2} \in \phi^{-1}\left(y_{1}\right)$. Then $y_{1} \neq x_{i}$ by definition, and hence $y_{2} \neq y_{1}$ also. Moreover, $y_{2} \neq x_{i}$, because if $y_{2}=x_{i}$, then taking $\phi$ of both gives $y_{1}=x_{i}$. Let $h$ be the unique map in $\operatorname{PGL}(2, \bar{K})$ with $h\left(x_{i}\right)=0, h\left(y_{1}\right)=1$, and $h\left(y_{2}\right)=\infty$, and let $\psi:=h \circ \phi \circ h^{-1}$ If $\psi$ has good reduction, then $\phi$ has potentially good reduction, and we are done.

Conversely, if $\phi$ has potentially good reduction, there is some $\tilde{h} \in$ $\operatorname{PGL}(2, \bar{K})$ such that $\tilde{\psi}:=\tilde{h} \circ \phi \circ \tilde{h}^{-1}$ has good reduction. By Lemma 2.3, the indifferent fixed point $\tilde{h}\left(x_{i}\right)$ of $\tilde{\psi}$ and its preimage $\tilde{h}\left(y_{1}\right)$ (under $\tilde{\psi}$ ) have different reductions in $\mathbb{P}^{1}(\bar{k})$. Since $\tilde{\psi}$ respects reduction, we must have $\overline{\tilde{\psi}}\left(\overline{\tilde{h}\left(y_{2}\right)}\right)=\overline{\tilde{h}\left(y_{1}\right)}$, and for the same reasons as in the previous paragraph, the reductions $\tilde{h}\left(x_{i}\right), \tilde{h}\left(y_{1}\right)$, and $\tilde{h}\left(y_{2}\right)$ must all be distinct points in $\mathbb{P}^{1}(\bar{k})$. Thus, there is some map $r \in \operatorname{PGL}\left(2, \mathcal{O}_{\bar{K}}\right)$ such that

$$
r\left(\tilde{h}\left(x_{i}\right)\right)=0, \quad r\left(\tilde{h}\left(y_{1}\right)\right)=1, \quad \text { and } \quad r\left(\tilde{h}\left(y_{2}\right)\right)=\infty .
$$

Then we must have $r \circ \tilde{h}=h$, and $\psi=r \circ \tilde{\psi} \circ r^{-1}$. Since $\tilde{\psi}, r$, and $r^{-1}$ all have good reduction, it follows that $\psi$ also has good reduction.

Case 2. The remaining case is that no $x_{i}$ is repelling or indifferent, and hence they are all attracting. That is, $\left|\lambda_{i}\right|_{v}<1$ for all $i$. Then no $\lambda_{i}$ is 1 , and hence the $d+1$ fixed points all have multiplicity 1 and therefore are distinct. In particular, $x_{1}, x_{2}$, and $x_{3}$ are all distinct. Let $h$ be the unique map in $\operatorname{PGL}(2, \bar{K})$ with $h\left(x_{1}\right)=0, h\left(x_{2}\right)=1$, and $h\left(x_{3}\right)=\infty$, and let $\psi:=h \circ \phi \circ h^{-1}$. If $\psi$ has good reduction, then $\phi$ has potentially good reduction, and we are done.

Conversely, if $\phi$ has potentially good reduction, there is some $\tilde{h} \in$ $\operatorname{PGL}(2, \bar{K})$ such that $\tilde{\psi}:=\tilde{h} \circ \phi \circ \tilde{h}^{-1}$ has good reduction. By Lemma 2.2, the attracting fixed points $\tilde{h}\left(x_{1}\right), \tilde{h}\left(x_{2}\right)$, and $\tilde{h}\left(x_{3}\right)$ of $\tilde{\psi}$ all have different reductions in $\mathbb{P}^{1}(\bar{k})$. Thus, there is some map $r \in \operatorname{PGL}\left(2, \mathcal{O}_{\bar{K}}\right)$ 
such that

$$
r\left(\tilde{h}\left(x_{1}\right)\right)=0, \quad r\left(\tilde{h}\left(x_{2}\right)\right)=1, \quad \text { and } \quad r\left(\tilde{h}\left(x_{3}\right)\right)=\infty .
$$

Then we must have $r \circ \tilde{h}=h$, and $\psi=r \circ \tilde{\psi} \circ r^{-1}$. Since $\tilde{\psi}, r$, and $r^{-1}$ all have good reduction, it follows that $\psi$ also has good reduction.

Acknowledgements. The author thanks Xander Faber and Joseph Silverman for their helpful suggestions. The author also gratefully acknowledges the support of NSF grant DMS-1201341.

\section{REFERENCES}

[1] Matthew H. Baker and Robert S. Rumely, Potential Theory and Dynamics on the Berkovich Projective Line, Amer. Math. Soc., Providence, 2010.

[2] R. Benedetto, Reduction, dynamics, and Julia sets of rational functions, $J$. Number Theory 86 (2001), 175-195.

[3] R. Benedetto, Attaining potentially good reduction in arithmetic dynamics, in preparation.

[4] P. Morton and J. Silverman, Rational periodic points of rational functions, Inter. Math. Res. Notices 2 (1994), 97-110.

[5] J. Rivera-Letelier, Dynamique des fonctions rationnelles sur des corps locaux, Astérisque 287 (2003), 147-230.

[6] R. Rumely, The minimal resultant locus, preprint. Available at arXiv:1304.1201.

Amherst College, Amherst, MA 01002

E-mail address: rlb@math.amherst.edu 